\title{
Mitología e intertextualidad en el Canto XXVI del Inferno de La Divina Comedia
}

\author{
Adrián Pignataro López \\ Escuela de Ciencias Políticas \\ Universidad de Costa Rica
}

\begin{abstract}
Resumen
La Divina Comedia de Dante Alighieri es una enciclopedia de la cultura clásica, que demuestra la relevancia de la Antigüedad en la Edad Media. El artículo analiza intertextos clásicos y elementos mitológicos -con base en conceptos de Mircea Eliade- en el Canto XXVI, en el cual se relata una historia del héroe griego Ulises. Finalmente, se muestra la continuidad del mito con dos ejemplos de la narrativa moderna.
\end{abstract}

Palabras claves: Dante Alighieri, La Divina Comedia, Edad Media, intertextualidad, mito

\begin{abstract}
Dante's Divine Comedy is an encyclopedia of classical culture, which demonstrates the importance of Antiquity on the Middle Ages. The paper analyzes the classical intertexts and the mythological elements - based on Mircea Eliade's concepts - on Canto XXVI, which tells the story of the Greek hero Ulysses. Finally, we show the continuity of the myth using two examples of modern narrative.
\end{abstract}

Key words: Dante Alighieri, Divine Comedy, Middle Ages, intertextuality, myth 


\section{Introducción}

$\mathrm{E}$ l artículo tiene como objetivo analizar elementos mitológicos e intertextuales presentes en la obra de Dante Alighieri $L a$ Divina Comedia, específicamente en su Canto XXVI del Inferno. Se busca demostrar que los mitos de dicho canto provienen de dos fuentes culturales: la antigüedad y el cristianismo. El énfasis se hará en la primera de ellas, es decir, la mitología clásica: griega y romana. Con esto se explicará que los mitos clásicos son intertextos de obras de la Antigüedad, principalmente de la Odisea y la Eneida.

Adicionalmente, se busca aportar evidencia sobre el continuismo del clasicismo entre la Antigüedad y el Renacimiento, a través de la Edad Media. Por lo que se sostiene que la cultura clásica -incluida su mitología- no muere con la caída del Imperio Romano, sino que se mantiene gracias al pensamiento medieval. En este sentido, se intenta refutar las acusaciones que ha recibido el periodo del Medioevo de ser una época oscura, donde el cristianismo se impone a la cultura pagana y la elimina; cuando en realidad ambas se articulaban y se sobreponían, como queda resaltado por Dante en su obra.

Finalmente, se resalta el valor intertextual del mito de Ulises, que pasa de Homero en la Antigüedad a Dante en la Edad Media e inclusive al siglo $\mathrm{XX}$, gracias a las obras de James Joyce y Primo Levi.

\section{Contexto histórico y cultural}

El estudio de la Edad Media ha estado plagado de falsedades, en especial de aquellas que la tildan de oscura y de incivilizada; una época en la que según algunos desaparece el legado clásico de las culturas griega y romana, aduciendo que no es descubierto sino hasta el Renacimiento del siglo XV (Mitre, 1976).

Algunos textos pretenden apoyar esa visión, que no pertenece únicamente a la opinión del público general sino también a la de algunos estudiosos; por ejemplo, en Ayala (2000, p. 5) -obra sobre mitos y leyendas medievales- se afirma que la Edad Media "fue una época de oscurantismo por encima de todo, plagada de leyendas y supersticiones, aunque también tuvo momentos de gran claridad".

El filólogo Manuel Antonio Quirós (2007, pp. 6-7) ha analizado las razones por las cuales la Edad Media ha sido objeto de estas valoraciones negativas. En primer lugar, ha contribuido el calificativo "media", creado durante el Renacimiento italiano y enfatizado por la Ilustración francesa. Esta última especialmente resaltaba los aspectos más irracionales de la Edad Media: alquimia, supersticiones, brujería, magia, astrología, inquisición, ignorancia y obediencia ciega a la Iglesia. Es decir, el discurso ilustrado creó una serie de generalizaciones falaces para criticar a la Iglesia católica y por extensión el periodo histórico en que ella fue dominante. Además, con la Revolución científica se viene a resaltar el carácter seudocientífico de los estudios medievales, ignorando así sus aportes a la civilización occidental.

Los ilustrados olvidan, por ejemplo, que la universidad es una institución claramente medieval, fundada luego de que el III Concilio de Letrán estipuló que "toda catedral organice un studium mediante la apertura de la escuela", con lo 
cual se establecen paralelamente corporaciones autónomas e independientes de maestros y alumnos llamadas universitates studiorum, que no obedecían a la jerarquía eclesiástica (Quirós, 2007, pp. 51-52).

En el aspecto filológico, se debe recordar que en la época medieval no solo se conservaron numerosos textos antiguos gracias a los copistas, sino que se produjo una enorme cantidad de literatura: 18000 textos conservados, mientras que se poseen tan solo 500 de la literatura clásica antigua (Quirós, 2007, p. 74).

Lo anterior no debe llevar a una lectura apologética de la Edad Media, sino proponer su estudio de manera objetiva y científica, para contrarrestar ese fenómeno llamado por Umberto Eco (2002, p. 293) como la "fuerza del falso", que según el semiólogo italiano ha sido motor de muchos eventos en la historia. ${ }^{1}$

Otras interpretaciones engañosas de la Edad Media dan cuenta del carácter oscuro, no en el plano ideológico sino en el estético. Sin embargo, el Medioevo identificaba la belleza con la proporción, la luz y el color, como muestran los vitrales góticos en la arquitectura y el Paradiso de Dante en la literatura. (Eco 2002, pp. 23-29)

En cuanto a la influencia de la cultura clásica es necesario hacer ciertas precisiones. La antigüedad es uno de los tres pilares culturales sobre los que se basa la Edad Media, junto con el cristianismo y el germanismo (Bühler, 2006). En este trabajo, se toma la posición del continuismo por la cual el Medioevo es la continuación de la tradición clásica grecorromana fusionada con el cristianismo (Quirós, 2007, p. 14).
$\mathrm{Al}$ respecto, advierte Quirós (2007, p. 60):

Cultura Clásica-Cristianismo casi siempre iban de la mano durante la Edad Media: imposible hablar de una sin hablar del otro. Incluso en su religiosidad, la Edad Media le es deudora a la Antigüedad Romana: desde la fundación de Roma, el romano siempre se mostró muy piadoso y religioso [...] por lo cual, era y es natural que el Cristianismo Romano se haya enriquecido con una enorme cantidad de elementos de la Roma pagana.

En esta cita se aprecia el legado de los valores religiosos romanos; no es, sin embargo, el único aporte de Roma y su cultura. Se destaca también la importancia de la filosofía griega -especialmente, Platón y Aristóteles- en la filosofía escolástica, el valor filológico de los textos clásicos y la importancia lingüística del latín y de su alfabeto (Quirós, 2007). Claramente, en otros aspectos, se observa un clivaje entre lo antiguo y lo medieval, en especial en la organización sociopolítica (el imperio da paso a los reinos feudales) y las instituciones hegemónicas (el Emperador y las magistraturas romanas son reemplazados por el papado y las autoridades de la Iglesia católica).

De modo que el clasicismo se encuentra presente durante todo el periodo medieval, aunque muchas veces de manera "escondida, mediante símbolos y alegorías, en la concepción cristiana", pero siendo de todos modos "dominante de toda la cultura medieval, aunque dominada por la espiritualidad cristiana" (Quirós, 2007, pp. 292-293).

El aspecto quizás más problemático consiste en definir en qué consistiría 
culturalmente la ruptura entre la Edad Media y el Renacimiento del siglo XV. ${ }^{2}$ Por la naturaleza del trabajo, es claro que el énfasis se hará en el sistema cultural; por lo tanto, se deben obviar los evidentes contrastes en lo político, económico y social entre ambas etapas. Sin embargo, vale destacar que en el Renacimiento italiano nacen los estados como unidades políticas consolidadas, se establece el mercantilismo como sistema económico y aparece un nuevo estrato social que comienza a ganar fuerza y poder: la burguesía (Ferrer y Cañuelo, 2002, p. 75).

Como cambio cultural, Jacob Burckhardt (1999 [1860], p. 97) ha señalado que el Renacimiento italiano se entiende como un retorno a la Antigüedad "en gran escala y de una manera general y decidida", que requería como condiciones necesarias la vida urbana, la convivencia e igualdad entre nobles y ciudadanos, y la necesidad de la cultura, teniendo el tiempo y los medios para satisfacerla.

Sin embargo, no queda claro cómo existe un retorno a la Antigüedad, cuando se afirmó que la Edad Media estuvo dominada por lo clásico. Tampoco resulta fácil diferenciar en literatura ambas etapas, como declara Bühler (2006, p. 207), puesto que los temas prevalentes siguen siendo los medievales incluso en los siglos XIV y XV.

Ferrer y Cuñuelo proponen que el Renacimiento está caracterizado por:

una revalorización de la Antigüedad grecolatina y un cuestionamiento de la civilización cristina [...] La nueva mirada hacia la Antigüedad y su cultura transformó el pensamiento y la función del arte medieval, dotándolo de un talante individualista y liberal [...] La exaltación de la individualidad colocó al artista en un primer plano, y el Humanismo, que sustituyó el teocentrismo de la Edad Media por el antropocentrismo, puso al hombre en el centro de la existencia. (2002, p. 75)

Del texto citado se desprenden conceptos importantes para entender el Renacimiento, debido a que por medio del humanismo se reinterpreta la Antigüedad desde una visión individualista y antropocéntrica (y, puede añadirse, sexista). Con esto se quiere aclarar que hay una continuidad de la cultura clásica desde la caída del imperio romano, a través de la Edad Media y hasta el Renacimiento del siglo XV. El clasicismo nunca desaparece, simplemente se interpreta de manera distinta en cada época: desde el cristianismo en la Edad Media y desde el Humanismo en el Renacimiento. ${ }^{3}$

En conclusión, se quiere rescatar el hecho de que la cultura clásica no desaparece con la caída del Imperium Romanum Occidentale: permanece vigente durante el Medioevo, combinada con el pensamiento cristiano hasta que es reinterpretada por el Humanismo en el siglo XV. De modo que se propone una visión de la Edad Media que contradice los estereotipos falsos motivados por la Ilustración y por los renacentistas; estos últimos existieron precisamente gracias a la conservación de los textos latinos y griegos durante la Edad Media.

\section{Dante Alighieri}

Sobre la vida de Dante Alighieri mucho se ha escrito y comentado, por lo que se pretende únicamente resumir los aspectos biográficos más importantes, así como recordar algunos de sus 
principales libros. Un aspecto interesante es que muchos de los datos sobre su vida provienen de su propia obra literaria, en especial de los versos de $L a$ Divina Comedia. ${ }^{4}$

Dante nació el 14 de mayo de 1265 en Florencia, con el nombre de Durante Cacciaguida Alighieri (Dante es pues un apócope). Provenía de una familia de buena posición económica: su padre -Alighiero II di Bellincioni d'Alighierose dedicaba a las finanzas. Teniendo 13 años, quedó huérfano al morir su madre Gabriella Abafi; su padre se casó por segunda vez (Mares, 2002, pp. 15-16).

Estudió retórica, literatura y lenguas bajo la tutela de Brunetto Latini. Continuó sus estudios en las artes liberales (trivium y quadrivium), aprendiendo el conocimiento general de la época, pero complementándolo con lecturas sobre filosofía y política. En la Universidad de Bologna se cultivó en derecho civil, derecho canónico, teología, filosofía moral, filosofía natural, ciencias físicas y medicina. Sus biógrafos afirman que Dante "aprendió durante su juventud todo lo que en aquellos tiempos se podía saber", tanto por su talento y dedicación como por contar con las mejores condiciones educativas (Mares, 2002, pp. 20-21).

Son numerosas las vicisitudes de su vida, pues se vio inmerso en los conflictos comunes entre facciones de Italia, ya sea entre güelfos y gibelinos como entre blancos y negros (división del partido güelfo). Tomó parte en la batalla de Campaldino y además fungió en puestos públicos de su ciudad. Los conflictos políticos le valieron el exilio de Florencia, por lo que muere en Ravenna la noche del 13 al 14 de septiembre del año 1321 (Mares, 2002).

Para el análisis literario de La Divina Comedia, resulta muy relevante recordar el gran amor de Dante: Beatrice. En palabras de Jorge Luis Borges, "Dante edificó el mejor libro que la literatura ha alcanzado para intercalar algunos encuentros con la irrecuperable Beatriz" (Borges, 1999, p. 95).

Dante, teniendo nueve años, conoció a Beatrice Portinari para amarla y recordarla por el resto de su vida, siendo su primer contacto casi una "experiencia mística"; es, sin embargo, trágico para Dante no solo el matrimonio de Beatrice con otro hombre sino principalmente la muerte temprana de ella en el año 1290. Con ello el poeta refuerza la idealización que hace de la mujer, siendo su amor un hecho sobrenatural y poético (Mares, 2002, pp. 29-34).

Beatrice es también un personaje literario presente en dos obras de Dante: Vita Nuova y Divina Commedia. Es decir, es una figura elaborada poéticamente e inspirada en la Beatrice histórica, por la cual el yo lírico expresará una pasión probablemente igual a la que sentía el Dante de carne y hueso. Resulta claro este amor divinizado de Dante cuando en Vita Nuova prometió escribir "lo que ningún hombre había escrito jamás de mujer alguna" (Ferro, 1990, p. 26). Pero es en los cantos del $P a$ radiso donde su amada alcanza el máximo nivel de adoración y divinización.

Otra obra importante de Dante es De monarchia, escrita en latín, en la cual defiende la independencia del imperio contra el papa (una controversia frecuente durante la Edad Media). Además, demuestra una fuerte influencia aristotélica al exponer su teoría social, similar a la expuesta por Santo Tomás de Aquino (Sabine, 1994, pp. 211-214).

También resulta relevante el tratado lingüístico De vulgari eloquentia, donde establece su tipología de las lenguas 
europeas y se propone buscar un "vulgar ilustre", analizando las distintas variedades del vulgar italiano para lograr encontrar una lengua nacional que responda a sus exigencias: pureza y nobleza. Aspiraba, por lo tanto, a encontrar una koiné italiana -que es precisamente la variedad florentinaque le serviría para escribir La Divina Commedia (Quirós, 2001, pp. 64-66).

En síntesis, Dante fue un hombre medieval que recibió una excelente educación, tanto clásica como teológica, cuya erudición es expuesta magistralmente en La Divina Comedia. Pero fue además un hombre con pasiones profundas, no solo por la teología y la divinidad sino por una mujer que se convertiría en uno de los personajes principales de su poema. En este punto, procede sintetizar la gran obra literaria de Dante, de la cual se extrajo un canto para su análisis mitológico.

\section{La Divina Comedia}

Dante comenzó la escritura de su gran poema en el año 1303 y lo finalizó poco antes de su muerte en 1321. Originalmente fue llamada Commedia, pero le fue añadido el adjetivo "divina" por Boccaccio, uno de los tantos comentaristas de la obra dantesca (Ferrer y Cañuelo, 2002, p.77). A continuación, se ofrece un breve examen de la obra según los elementos retóricos clásicos (Quirós, 2004, p. 281): inventio (el hallazgo de las ideas desarrolladas), dispositio (el ordenamiento de las ideas encontradas) y elocutio (el buen estilo empleado en las anteriores).

$\mathrm{Su}$ inventio es imposible de sintetizar en pocas líneas al tratarse de una obra tan extensa, completa y enciclopédica. Mas para dar claridad a este trabajo se recordará que el poema narra en primera persona el viaje del poeta (Dante) en los reinos ultraterrenales Infierno, Purgatorio y Paraíso, comenzando la travesía el 25 de marzo o el 8 de abril de 1300 - según sus comentaristas - y durando siete días (Ferro, 1990, p. 29). En su marcha estará acompañado por dos guías: el poeta Publio Virgilio Marón y su amada Beatrice Portinari. Su travesía lo hace conocer no solo las regiones de castigo, penitencia y gloria, sino los personajes míticos, legendarios e históricos que en ellas se encuentran. Santos, papas, filósofos, poetas, guerreros y almas en general constituyen la plétora de personajes secundarios que revelan a Dante cómo funciona la justicia divina y las leyes naturales que rigen estos mundos. De modo que la inventio presente es sumamente creativa y vasta: "es un admirable tratado de filosofía, de teología, de astronomía, de mitología, de moral, de hagiografía" (Alarcón, 1998, p. 13).

De la dispositio se puede decir que la obra está estructurada en tres cánticas, a las que les corresponde una región del más allá y de ahí toman sus nombre: Inferno, Purgatorio y Paradiso. Cada una de las cánticas está conformada por 33 cantos, excepto la primera que posee uno más, el cual funciona como preámbulo de la obra. En total, la Commedia está compuesta por 14.233 versos, organizados en tercetas de rima alternada (Ferro, 1990, p. 29).

La elocutio de La Divina Comedia demuestra un valor literario inconmensurable; es un libro tan perfecto en el que "no hay palabra injustificada" (Borges, 1999, p. 9). La pulcritud y belleza de sus versos la han convertido en uno de 
los grandes clásicos no solo de la literatura italiana sino también de la literatura occidental y románica.

Además de su trascendencia literaria, la Commedia constituye una obra lingüística fundamental para la formación de la lengua nacional italiana. En esta obra Dante pone en práctica su teoría expuesta en De vulgari eloquentia al escribirla no en latín sino en una lengua neorrománica, que según él representaba la mejor variedad de las existentes en Italia. Entiéndase que el latín vulgar produjo durante la Edad Media una plétora de lenguas en la península itálica; Dante escoge la variedad toscana -particularmente la florentina- como "vulgar ilustre" para escribir la Commedia. Gracias a los méritos de este poeta -junto con los propios de otros autores como Petrarca y Boccaccio- y por medio de ciertos factores políticos particulares, la variedad florentina se establece como lengua nacional (proceso de koineización). De este modo, esta lengua se transformaría a través de los años hasta llegar a ser el italiano contemporáneo (Quirós, 2001). ${ }^{5}$

Aunque algunos pretendan ubicar La Divina Comedia como una obra moderna -por ejemplo, Ferrer y Cañuelo (2002) la catalogan en el Renacimiento-, se considera acá primordialmente medieval, compartiendo en este sentido el criterio de Bühler (2006, p. 206), porque sus dos grandes fuentes literarias son las dos principales bases culturales de la Edad Media: el clasicismo y el cristianismo. Estas son asimismo representadas respectivamente por los guías de Dante -protagonista- en su viaje: Virgilio y Beatrice. El primero se considera símbolo de la razón y la segunda de la teología, con sus textos respectivos, la Eneida y la Biblia. Por lo que el simbolismo en la Commedia es complejo: es necesario tener una profunda erudición del pensamiento medieval para descifrarlo, especialmente en sus fuentes clásicas y cristianas.

Incluso algunos textos hablan de la presencia de símbolos cabalísticos en La Divina Comedia, especialmente en lo que se refiere a la importancia del número tres y de su múltiplo nueve. Se sugiere, por ende, que Dante fue iniciado en el estudio de la cábala (Menué, 2004, pp. 161-162). Sin embargo, resulta cuestionable aceptar que un autor tan firmemente cristiano, que rechaza todo aspecto infiel y sitúa a Mahoma bajo tormento en el Infierno (Canto XXVIII), pudiese abrazar un culto esotérico-judaico (es decir, no cristiano) como es la cábala. En todo caso, para entender el simbolismo numérico del tres no es necesario remitirse a los estudios herméticos, puesto que el cristianismo aporta sus propios significados a esta cifra: la Trinidad, la resurrección al tercer día, las tres tentaciones en el desierto, entre otros.

En cuanto a la mitología presente, se quiere rescatar algunos elementos de análisis de la obra en general, sin entrar en detalles sobre mitos y hierofanías, reservados para la siguiente sección.

En primer lugar, examínese la relación entre espacio sagrado y espacio profano, conceptos elaborados por Mircea Eliade en su fenomenología de las religiones. Al examinar La Divina Comedia tan solo el adjetivo añadido por Boccaccio ofrece un indicio sobre la naturaleza sagrada del poema. Aunque no se trata propiamente de un texto sagrado, es decir, digno de culto ritual y por medio del cual un ser humano se transporta de lo profano a lo sagrado, como lo es la Torah, los Vedas, el Corán 
o los evangelios, su historia se desarrolla en un espacio sagrado. Inclusive el mismo texto lo autoafirma en el Paradiso: poema sacro (Canto XXV, v. 1). ${ }^{6}$

Se podría objetar el hecho de que toda la obra se desarrolle en un espacio sagrado, alegando que este se identifique únicamente para los cantos en el Paraíso, mientras que el Infierno y el Purgatorio son profanos por la ausencia directa de lo divino y la presencia de pecadores. Es cierto que lo pagano en la Edad Media era considerado profano, mientras que lo sagrado es aquello cristiano (Quirós, 2007, p. 29); no obstante, en este trabajo, se propone que el texto logra consagrar ese mundo pagano, romano y precristiano a partir de las hierofanías que se presentan a lo largo del poema. Dante logra sacralizar aquellos elementos profanos comunes de la vita cotidiana: los pecados, la vida social, la política, la corrupción, su ciudad Florencia, el amor de Paolo y Francesca, el mundo Antiguo...

Las regiones del Infierno y del Purgatorio son purificaciones espirituales para Dante, necesarias para expiar sus pecados y poder ser digno de llegar al Paraíso y contemplar a la Divinidad. Estos ritos iniciáticos son, según Eliade (1973, p. 164), importantes en el mito heroico y en las mitologías de la muerte, incluyendo algunos el ser engullido por parte de un monstruo (en este caso, las cavernas del Infierno). Además: "el acceso a la vida espiritual comporta siempre la muerte para la condición profana, seguida de un nuevo nacimiento" (Eliade, 1973, p. 169). Por este motivo, cuando Dante decide seguir a Virgilio hacia las regiones de ultratumba (Inferno, I, vv. 130-136), da inicio a un ritual de purificación -que incluye su inmersión en los ríos Letè y Eunoè (Purgatorio, Cantos XXXI y XXXIII) y abandonando así el espacio profano con el propósito de ser digno de llegar a contemplar a Dios y al Amor que mueve el sol y las estrellas (Paradiso, Canto XXXIII).

Otro elemento conceptual de Eliade que es reconocible sin dificultad en Dante es el axis mundi. Afirma el mitólogo que "la comunicación se expresa a veces con la imagen de una columna universal, que une, a la vez que lo sostiene, el Cielo con la Tierra, y cuya base está hundida en el mundo de abajo" (Eliade, 1973, p. 38). La topografía dantesca es una representación exacta de ese axis mundi: comienza en la montaña de Sión por donde Dante entra al Infierno, teniendo este forma de cono invertido, y finaliza en el centro de la tierra donde yace Lucifer; la línea continúa hasta el monte del Purgatorio (en posición geográfica opuesta al monte de Sión) y apunta hacia los distintos cielos, y une, por lo tanto, las tres zonas cósmicas.

\section{El Canto XXVI del Inferno}

El argumento del Canto XXVI es el siguiente: Dante y su guía Virgilio, en su viaje por el Infierno, se encuentran primero con cinco ladrones florentinos. Seguidamente pasan al octavo foso del octavo círculo, donde ven arder a los consejeros fraudulentos y topan con Ulises y Diomedes. A petición de Dante, el primero de los héroes narra su última travesía, que reza así: en vez de regresar a Ítaca como cantó Homero, Ulises siguió navegando hacia el oeste con sus compañeros hasta que divisó una montaña enorme 
y su barca fue consumida por el agua, hallando él y su tripulación la muerte.

Ahora se procede a analizar con detalle los mitos, hierofanías e intertextos del Canto, tres elementos que están fuertemente relacionados entre sí. En otras palabras, generalmente donde hay un mito está también un intertexto o una hierofanía. ${ }^{7}$

En primer lugar, hay que señalar que existen mitos identificables en el Canto XXVI que se evidencian a su vez en la totalidad del poema. En este sentido, se recuerdan el mito escatológico y el mito heroico. El primero se refiere a la vida después de la muerte, o bien a la vida en ultratumba. Dante naturalmente desciende a los infiernos y recorre las regiones del más allá en un proceso de imitatio et aemulatio de Eneas y San Pablo (como se podía esperar, uno pagano y otro cristiano, con lo que se perpetúa este binomio ya señalado entre lo clásico-medieval). Sin embargo, Dante admite que, pese al parangón, no es digno de ese honor ("Io non Enëa, io non Paulo sono;/me degno a ciò né io né altri 'l crede" Inferno, Can. II, vv. 32-33).

El mito escatológico clásico se encuentra en la obra de Publio Virgilio Marón, la Eneida. Recuérdese que Virgilio es el primer guía de Dante, considerado por este como el más grande poeta, maestro y el arquetipo de la cultura clásica latina (Inferno, I, vv. 79-87). De modo que le hace homenaje tanto como figura histórica y literaria como a su obra principal, la Eneida.

En la obra de Virgilio, Eneas -héroe troyano que escapó en el saqueo de Ilión y de quien se origina la estirpe romanadesciende a los infiernos para hablar con el espíritu de su padre (en el Libro Sexto de la Eneida). Hay notables similitudes entre ambos textos, como por ejemplo:
- La presencia de Caronte, barquero en el río Aqueronte (Inferno, III), de Minos (Inferno, V) y de Plutón (Inferno, VII).

- La existencia de un guía: Virgilio en el Infierno para Dante y la Sibila de Cumas para Eneas.

- El requerimiento de rituales para poder entrar a las regiones de ultratumba. Eneas debió llevarle una rama de oro a Proserpina y Dante tuvo que pasar los ríos Letè y Eunoè.

- Los castigos para los malos y la recompensa para los puros. En la Eneida, no existe el Paraíso como lo concibe Dante y la teología medieval, pero posee dos regiones infernales según el destino de cada espíritu: "Este es el sitio en que el camino se divide en dos partes: la de la derecha se dirige al palacio del poderoso Plutón, es la senda que nos llevará a los Campos Elíseos; la de la izquierda conduce al impío río Tártaro, donde los malos sufren su castigo" (Eneida, Libro Sexto).

En cuanto a la escatología cristiana, el apóstol Pablo revela que ascendió al tercer cielo sin saber si en cuerpo o en alma (Segunda Epístola a los Corintios, XII, 1-2). Dante también llegará a los cielos y al igual que Pablo está en la presencia mística de la Divinidad.

Otros investigadores han destacado la influencia que tuvo la escatología musulmana en Dante Alighieri y su obra, por ejemplo: los estudios de Miguel Asín Palacios, Enrico Cerulli y Maria Corti. La tesis señala que, así como los árabes introdujeron a Aristóteles en Occidente durante la Edad Media, sus textos escatológicos sobre los viajes de Mahoma al Infierno y al Paraíso fueron también fuentes directas para Dante 
(Alarcón, 1998, pp. 14-17 y Borzi, 2009, p. 21). Estas investigaciones han dado resultados bastante sólidos sobre la intertextualidad musulmana en $L a$ Divina Comedia.

Como mito heroico, tanto Eneas como Dante son héroes en sus propias historias. El primero en su misión de fundar la estirpe romana y el segundo para huir de las tres fieras que le impedían el paso (simbólicamente, sus pecados) y realizar el sagrado viaje para conocer la Divinidad. Ambos viajes heroicos son predestinados por dioses: Júpiter y Dios, respectivamente. Además, cuentan con pruebas de coraje y espíritu, componentes básicos de los viajes de los héroes (Campbell, 1959, p. 94).

Se presencian mitos específicos para el Canto XXVI. Cuando Dante se acerca a las llamas del octavo foso, recuerda el ascenso al cielo del profeta Elías en un carro de fuego (Inferno, XXVI, vv. 34-42). Este mito (o dogma cristiano, según el punto de vista) fue relatado en el Libro Segundo de los Reyes (II, 11). Es claramente un mito escatológico porque se refiere al paso de la vida a la muerte del profeta; al mismo tiempo, es sobre todo una imponente hierofanía uránica que contiene al Yahvé hebreo que es sin duda un dios celestial pero también un mítico ascenso al cielo (de ahí lo uránico).

El Canto continúa mencionando otros personajes de la mitología griega como Etécles, Aquiles, Diomedes, Aquiles y Ulises (todos mitos heroicos). Se hará énfasis en este último por la importancia dada por Dante y por su valor intertextual.

A petición del viajero florentino, Ulises cuenta por qué está en la fosa pírica. Como se señaló anteriormente, el héroe homérico no volvió a su morada con
Penélope y Telémaco ni tampoco vertió sangre sobre los pretendientes de su mujer. En lugar de poder declarar que "yo sufriendo los infortunios de que me enviaron Zeus y los demás dioses para detenerme lejos de la patria cuando anhelaba volver a ella" (Odisea, Rapsodia XXIII), el Laertíada declara a Dante y a su guía que ni la dulzura de su hijo, ni la piedad de su padre ni el amor de Penélope lo hicieron volver a su país debido a su ambición y ardor por conocer el mundo y el mar (Canto XXVI, vv. 94-102).

De modo que Dante toma el mito de Homero como un intertexto para contar un nuevo final para el viaje de Ulises: el héroe al pasar las columnas de Hércules siguiendo el mar le aparece una montaña más alta que cualquiera que haya visto (según los comentaristas, la montaña del Paraíso terrestre) hasta que las aguas hicieron girar la barca tres veces para luego cerrarse sobre ellos (Inferno, XXVI, vv. 108-142).

Esta hierofanía acuática representa las leyes naturales que impiden a un pagano impuro llegar a un espacio sagrado como el Purgatorio (Getto et al., 1977, p. 68). Se sugiere la presencia de ciertos mitos, quizás de tipo cosmológico, que según su propio significado señalan el orden racional del universo, derivado de la voluntad divina que ni el propio héroe homérico logra transgredir.

La hierofanía acuática que revela el final dantesco de Ulises trae consigo un simbolismo propio; según Eliade (1986, p. 178), representa una muerte y resurrección, así como una regresión preformal, una regeneración total y un nuevo nacimiento. Ulises, según Dante, muere para nacer en el mundo escatológico construido por él, se transfigura en fuego como símbolo de su coraje pero también como castigo 
del pecado. Se podría decir que el poeta "regenera" a Ulises en su Canto, lo transporta de la antigua Grecia al Medioevo precisamente por medio de la intertextualidad literaria.

Finalmente, se quiere recordar una potente frase del Ulises de Dante, cuando pronuncia a su tripulación sobre la justificación de su viaje:

Considerate la vostra semenza:

fatti non foste a viver come bruti, ma per seguir virtute e canoscenza (Inferno, XXVI, vv. 118-120).

Considerad vuestro origen:

no habéis sido creados para vivir como

[brutos,

sino para seguir virtud y conocimiento [traducción del autor]

En estas palabras, está el ser humano creado para buscar la virtud y el conocimiento, la moral y la ciencia, la religión y la razón, Beatrice y Virgilio, el cristianismo y el clasicismo, lo medieval y lo antiguo, lo sagrado y lo profano, lo divino y lo pagano... Con un verso, Dante resume sus cien cantos.

\section{Conclusiones}

La Divina Comedia de Dante Alighieri es una enciclopedia de las grandes fuentes que alimentaron la cultura en la Edad Media, es decir, los mundos clásico y cristiano. La obra demuestra que existe un puente que une el mundo antiguo con el Renacimiento del siglo XV. Los modernos no descubren ni a los griegos ni a los romanos; los reinterpretan desde el humanismo, por lo cual no se puede atribuir el epíteto de edad oscura al periodo medieval, por lo menos al argumentar una ignorancia del legado grecorromano.

Dante como escritor se nutrió de la mejor educación tanto clásica como medieval, al conocer profundamente la literatura y la filosofía antiguas en el mismo nivel que la teología. Ambos saberes se simbolizan en Virgilio y en Beatrice, los guías de Dante (como personaje ficticio del poema).

Pero la Commedia no es solamente un conjunto de conocimiento filosófico, histórico y teológico, sino también mitológico. Se demostró que muchos de los conceptos de Mircea Eliade -como axis mundi, ritos de purificación, hierofanías acuáticas y uránicas, mitos escatológicos y heroicos- desfilan ante los ojos del lector. Además, el texto de Dante es notablemente intertextual, citando y emulando a autores clásicos como Homero y Virgilio, además de las Escrituras. El Canto XXVI evidencia que el mito de Ulises no muere con el poema homérico: se revitaliza en la Edad Media.

Además, la mitología de Ulises tampoco acaba con Dante puesto que otros autores lo han retomado. Por ejemplo, el irlandés James Joyce, quien -a partir de su obra Ulysses (2000 [1922])reconstruye el mito de Odiseo para convertirlo en un antihéroe moderno: Leopold Bloom. Las equivalencias con la obra de Homero van desde el título mismo hasta la estructura narrativa, personajes y temas (Greenway, 1955; ver también Wykes, 1968).

Otro autor del siglo pasado que referencia el mito es el italiano Primo Levi, quien escribió su novela Se questo è un uomo, basada en su experiencia como prisionero de un campo de concentración alemán durante la Segunda Guerra Mundial. En un capítulo titulado "Il canto di Ulisse" 
(Levi, 2005 [1947], pp. 98-103), el autorpersonaje recuerda el Canto XXVI del Inferno, lo lee a sus compañeros del Lager y revive personalmente los versos dantescos en su propio dolor, miseria y sufrimiento, como las aguas que consumen y destruyen el viaje de Ulises.

En conclusión, la mitología clásica no desaparece nunca en la literatura y Ulises es un buen ejemplo de ello. Sea en un poema medieval, en el modernismo europeo o en el realismo de la posguerra, los mitos son perpetuados por medio de la palabra.

\section{Notas}

1. Por ejemplo, Eco (2002, pp. 295-303) recuerda la falsedad producida por el pensamiento del siglo XVIII que afirmaba que tanto los antiguos como los medievales pensaban que la Tierra era plana. A propósito del artículo, una prueba clara de dicha mentira se encuentra en la misma Commedia de Dante, en la cual su protagonista empieza el viaje en un hemisferio (entrada del Infierno) y lo continúa en el contrario (monte del Purgatorio), dando la idea de la redondez terrestre.

2. Se insiste en diferenciar el Renacimiento italiano del siglo XV (que ha adquirido el título de Renacimiento por antonomasia) para recordar la existencia de otros renacimientos anteriores, como el carolingio, el otoniano, el de Montecassino y el del siglo XII (cfr. Quirós, 2007, I sección).

3. El humanismo se encarga de "reivindicar el auténtico y verdadero valor de la cultura clásica según se manifestaba en sus textos, a los que se comenzaba a dar un tratamiento filológico no mediante las interpretaciones de los escolásticos de la Edad Media Tardía" (Quirós, 2001, p. 68). Tiene sus orígenes en el pensamiento filosófico antiguo, presente sobre todo en el Círculo de los Escipiones y en Cicerón; en el Renacimiento, sus cultivadores más emblemáticos son Francesco Petrarca y Erasmo de Rotterdam.

4. Por ejemplo, en La Divina Comedia, afirma que se encuentra "en el medio del camino de nuestra vida" (Inferno, I, v.1); en el Convivio, dice que la mitad de la vida son 35 años, puesto que según las escrituras la existencia humana dura 70 años. Sabiendo que su viaje imaginario en la Commedia se desarrolla en el 1300, año del Jubileo, entonces se resuelve que el año de su nacimiento es 1265 (Borzi, 2009, p. 7).

5. El italiano unificado se debe en parte al éxito literario de Dante, pero también a la prosa de otros escritores como Manzoni, Svevo y Moravia, y a los medios de comunicación televisivos (Eco, 2002, pp. 7-8). Por lo que Dante tiene el doble mérito de establecer una lengua literaria pero a la vez de contribuir a la consolidación nacional de una lengua hablada (aunque sin duda en perjuicio de otras lenguas italianas minoritarias y de variedades dialectales igualmente valiosas).

6. Se procurará citar La Divina Commedia según el canto y el verso, y no por número de página, para facilitar su consulta en cualquier edición italiana. Las traducciones en español -realizadas en prosa- solamente permiten encontrar el canto por número pero no los versos.

7. Estos conceptos se comprenden de la siguiente manera: por mito, se hace referencia a una "historia de lo acontecido in illo tempore" (Eliade, 1973, pp. 84-85). Mientras que hierofanía se 
entiende por etimología y por el mismo desarrollo conceptual que le dio Eliade: es una manifestación de lo sagrado (Eliade, 1973, p. 38). Finalmente, intertexto se define como "la relación entre el texto analizado y otros textos leídos o escuchados, que se evocan consciente o inconscientemente o que se citan, ya sea parcial o totalmente" (Beristáin, 1997, p. 269 citada por Quirós, 2007, p. 262).

\section{Bibliografía}

Alighieri, Dante. Divina Commedia. Roma: Newton Compton.

Alighieri, Dante. La Divina Comedia. Madrid: Edimat.

Alarcón, Benito (1998). Prólogo. En Dante Alighieri, La Divina Comedia. Madrid: Edimat.

Ayala, R.R. (2000). Mitos y leyendas de la Edad Media. Barcelona: Edicomunicación.

Borges, Jorge Luis (1999). Nueve ensayos dantescos. Madrid: Alianza.

Borzi, Italo (2009). "Dante Alighieri: profilo biografico" e Introduzzione. En Dante Alighieri, Divina Commedia. Roma: Newton Compton.

Burckhard, Jacob (1999) [1860]. La cultura del Renacimiento en Italia. México: Porrúa.

Bühler, Johannes (2006). La cultura en la Edad Media. Barcelona: Reditar Libros.

Campbell, Joseph. (1954). El héroe de las mil caras. Psicoanálisis del mito. México: Fondo de Cultura Económica.

Eco, Umberto (2002). Sulla letteratura. Milano: Bompiani.

Eliade, Mircea (1973). Lo sagrado y lo profano. Madrid: Guadarrama.
Eliade, Mircea (1986). Tratado de historia de las religiones. México: Era.

Ferrer, Jordi y Susana Cañuelo (2002). Historia de la Literatura Universal. Barcelona: Óptima.

Ferro, Federico (1990). Breve historia de la literatura italiana. México: Porrúa.

Getto, Giovanni et al. (1977). Storia della letteratura italiana. Milano: Rizzoli.

Greenway, John (1955). A Guide through James Joyce's Ulysses. College English, 17(2), 67-78.

Homero. La Odisea. Madrid: Alba.

Joyce, James (2000) [1922]. Ulysses. London: Penguin Books.

Levi, Primo (2005) [1947]. Se questo è un uomo. Torino: Einaudi.

Mares, Roberto. (2002). Dante. México: Editorial Tomo.

Menué, Konrad (2004). La masonería y las ciencias ocultas. Barcelona: Reditar Libros.

Mitre, Emilio (1976). Introducción a la historia de la Edad Media europea. Madrid: Istmo.

Quirós, Manuel Antonio (2001). Del latín al italiano. San José: Ediciones Sanabria.

Quirós, Manuel Antonio (2004). Ars litterarum o la conformación artística de un idioma. Revista de Filología, Lingüística y Literatura, 30 (2), 263-288.

Quirós, Manuel Antonio (2007). La Edad Media, los Carmina Burana y Carl Orff. San José: Editorial UCR.

Sabine, George H. (1994). Historia de la teoría política. México: Fondo de Cultura Económica.

VV.AA. Biblia de Jerusalén. Bilbao: Desclée de Brouwer.

Virgilio Marón, Publio. Eneida. Madrid: Edimat.

Wikes, David (1968). The Odyssey in Ulysses. Texas Studies in Literature and Language, 10 (2), 301-316. 
